\title{
Dissociable Intrinsic Connectivity Networks for Salience Processing and Executive Control
}

\author{
William W. Seeley, ${ }^{1}$ Vinod Menon, ${ }^{2,3}$ Alan F. Schatzberg, ${ }^{2}$ Jennifer Keller, ${ }^{2}$ Gary H. Glover, ${ }^{3,4}$ Heather Kenna, ${ }^{2}$ \\ Allan L. Reiss, ${ }^{2,3}$ and Michael D. Greicius ${ }^{2,5}$ \\ ${ }^{1}$ Department of Neurology, School of Medicine, University of California, San Francisco, San Francisco, California 94143, and ${ }^{2}$ Department of Psychiatry, \\ ${ }^{3}$ Program in Neuroscience, ${ }^{4}$ Department of Radiology, and ${ }^{5}$ Department of Neurology, Stanford University School of Medicine, Stanford, California 94305
}

\begin{abstract}
Variations in neural circuitry, inherited or acquired, may underlie important individual differences in thought, feeling, and action patterns. Here, we used task-free connectivity analyses to isolate and characterize two distinct networks typically coactivated during functional MRI tasks. We identified a "salience network," anchored by dorsal anterior cingulate (dACC) and orbital frontoinsular cortices with robust connectivity to subcortical and limbic structures, and an "executive-control network" that links dorsolateral frontal and parietal neocortices. These intrinsic connectivity networks showed dissociable correlations with functions measured outside the scanner. Prescan anxiety ratings correlated with intrinsic functional connectivity of the dACC node of the salience network, but with no region in the executive-control network, whereas executive task performance correlated with lateral parietal nodes of the executive-control network, but with no region in the salience network. Our findings suggest that task-free analysis of intrinsic connectivity networks may help elucidate the neural architectures that support fundamental aspects of human behavior.
\end{abstract}

Key words: fMRI; functional connectivity; anterior cingulate; insula; salience; anxiety

\section{Introduction}

Prevailing theories of brain function emphasize modularity and connectivity (Mesulam, 1998). Modularity describes specialized processing within dedicated brain regions, whereas connectivity relates to contemporaneous information flow across large-scale distributed networks. In nonhuman primates, modularity and connectivity data have been woven together through electrophysiological and tract tracing studies. These methods are difficult to implement in humans, creating a need for novel neuroimaging approaches.

In response, functional connectivity magnetic resonance imaging (fcMRI) studies have been used to detect brain regions whose blood oxygen level-dependent (BOLD) signal fluctuations correlate across time in task-free or "rest" settings (Biswal et al., 1995; Greicius et al., 2003; Beckmann et al., 2005; Fox et al., 2005). Using fcMRI, researchers have identified resting-state cortical networks presumed to underlie sensory, motor, and cognitive functions (Lowe et al., 1998; Cordes et al., 2000; Beckmann et al., 2005). These results support the intuitive notion that the "resting" brain is never truly resting. Rather, in the absence of an externally cued task, coherent brain activity can be demonstrated within a finite set of distributed spatial maps (Beckmann et al.,

Received Sept. 28, 2006; revised Jan. 19, 2007; accepted Jan. 22, 2007.

This work was supported by grants from the Larry L. Hillblom Foundation, Alzheimer's Association Grant NIRG04-1060, National Science Foundation Grant 0449927, and National Institutes of Health Grants K08 AG027086-01, HD047520, MH050604, MH019938, NS048302, RR000070, and RR009784.

Correspondence should be addressed to Michael D. Greicius, Departments of Neurology and Neurological Sciences and Psychiatry and Behavioral Sciences, Stanford University School of Medicine, 300 Pasteur Drive, Room A343, Stanford, CA 94305-5235. E-mail: greicius@stanford.edu.

DOI:10.1523/JNEUROSCI.5587-06.2007

Copyright $\odot 2007$ Society for Neuroscience $\quad$ 0270-6474/07/272349-08\$15.00/0
2005). Therefore, in an effort to avoid misconceptions evoked by "resting-state networks," we will apply the term intrinsic connectivity networks (ICNs) throughout this paper.

In contrast to fcMRI analyses that define networks based on intrinsic connectivity, most functional imaging studies employ cognitive subtraction paradigms. A key limitation to these paradigms is that they do not distinguish task-related activation in a single network from coactivation of distinct networks (Friston et al., 1996). Regions such as the dorsal anterior cingulate cortex (dACC), orbital frontoinsula (FI), lateral prefrontal cortex (PFC), and lateral parietal cortex are consistently recruited by cognitively demanding tasks and frequently interpreted as constituting a unitary network, which we refer to as the taskactivation ensemble (TAE). We favor the novel term TAE over task-activation network because the literature increasingly supports a separation of this TAE into at least two distinct subnetworks. Whereas lateral PFC and parietal regions are frequently coactivated with dACC and FI in tasks of attention, working memory, and response selection (Menon et al., 2001; Curtis and D’Esposito, 2003; Kerns et al., 2004; Ridderinkhof et al., 2004a), the dACC and FI also activate in response to pain, uncertainty, and other threats to homeostasis (Peyron et al., 2000; Craig, 2002; Grinband et al., 2006). These data suggest that dACC and FI are not responding in a task-specific manner but rather to a degree of personal salience, whether cognitive, homeostatic, or emotional, that cuts across tasks and requires changes in sympathetic tone (Critchley et al., 2004; Critchley, 2005).

In the current study, we pursued the following fundamental questions related to ICNs. Do regions activated as part of the TAE reflect distinct ICNs that support distinct subprocesses? Can ICNs, with their signature spatial and temporal profiles, be linked 
to emotional and cognitive functions? We addressed these questions by isolating two functional nodes from the TAE, deriving distinct ICNs associated with each, and demonstrating predictable correlations between these ICNs and individual differences in emotion and cognition measured outside the scanner. The results highlight the distributed regional architecture and functional relevance of two ICNs critical for adaptive human behavior.

\section{Materials and Methods}

\section{Subjects}

For the region of interest (ROI) analysis, 14 healthy subjects (ages 18-25; mean age, 21.2 years; SD, 2.2; seven female, all right-handed) were scanned after giving written informed consent. These subjects performed three functional tasks followed by a 4 min scan in which they were instructed only to keep their eyes closed and try to hold still. Results from the functional tasks have been published previously in a study comparing fMRI activation at different magnetic field strengths (Krasnow et al., 2003). These subjects were not asked to undergo neuropsychological testing or assessments of anxiety.

For the independent component analysis (ICA), 21 healthy subjects (ages 18-70; mean age, 34.6; SD, 15.8; 11 female, 20 right-handed) underwent a 5 min task-free scan after giving written informed consent. They were instructed only to keep their eyes closed and try to hold still. Three additional subjects were scanned but their data were excluded before analysis because of excessive motion and/or scanner artifact. Fifteen of the 21 subjects completed an anxiety rating scale just before entering the scanner. All 21 subjects underwent neuropsychological testing.

\section{Behavioral data acquisition}

Subjective anxiety rating. Just before entering the scanner, subjects were asked to rate their level of anxiety on a visual analog scale (VAS) ranging from 0 (none) to 10 (maximal). The range was $0-7$ with a mean of 1.8 and SD of 2.0.

Executive functioning. The Trail Making Test (TMT) examines the ability to switch between competing mental sets while performing a visuomotor search task. The TMT consists of a simple (part A) and a complex (part B) segment. Part A requires the rapid connection of 25 circled numbers randomly scattered on a page. Part B consists of circled numbers and letters $(1-13 ; \mathrm{A}-\mathrm{L})$ that must be connected in alternating sequence (i.e., 1-A-2-B-3-C, etc.). Successful Trails B performance requires working memory, mental flexibility, resistance to distraction, and rapid visual processing. Subjects are asked to complete the task as quickly as possible and their time and accuracy are recorded. Mean time for Trails B - A was $31.2 \mathrm{~s}$ (range, 1-74; SD, 17.5). During the Trails segment $B$, five subjects made one error and 16 subjects made no error.

\section{MRI data acquisition/preprocessing}

ROI data. Functional images were acquired on a 3T GE Signa Excite scanner (GE Medical Systems, Milwaukee, WI) using a standard GE whole head coil. Twenty-eight axial slices ( $4 \mathrm{~mm}$ thick, $0.5 \mathrm{~mm}$ skip) parallel to the plane connecting the anterior and posterior commissures and covering the whole brain were imaged using a $\mathrm{T} 2{ }^{\star}$ weighted gradient echo spiral pulse sequence (repetition time, $2000 \mathrm{~ms}$; echo time, $30 \mathrm{~ms}$; flip angle, $80^{\circ}$ and 1 interleave) (Glover and Lai, 1998). The field of view was $200 \times 200 \mathrm{~mm}^{2}$, and the matrix size was $64 \times 64$, giving an in-plane spatial resolution of $3.125 \mathrm{~mm}$. To reduce blurring and signal loss arising from field inhomogeneities, an automated high-order shimming method based on spiral acquisitions was used before acquiring functional MRI scans (Kim et al., 2000).

Data were preprocessed and analyzed using Statistical Parametric Mapping 99 (SPM99) (http://www.fil.ion.ucl.ac.uk/spm). SPM99 was used here because the ROIs used in the current study were derived from a previously published two-back working-memory dataset analyzed in SPM99 (Krasnow et al., 2003). Images were corrected for movement using least square minimization without higher-order corrections for spin history, and normalized (Friston et al., 1995) to the Montreal Neurological Institute (MNI) template. Images were then resampled every 2 $\mathrm{mm}$ using sinc interpolation and smoothed with a $4 \mathrm{~mm}$ Gaussian kernel to decrease spatial noise.

ICA data. Acquisition parameters for the ICA analysis were identical to those used in the ROI analysis. The task-free scan in this case was 5 min long. Preprocessing steps, including realignment, normalization, slice time correction, and smoothing as well as statistical analysis were performed using SPM2.

All functional data were overlayed on the MNI template available in MRIcro (http://www.sph.s.c.edu/comd/rorden/mricro.html) for presentation purposes.

\section{fMRI intrinsic connectivity network analyses}

ROI data. The two ROIs used in the initial phase of this study were derived from the functional map of a previously reported two-back working-memory task (for further details, see Krasnow et al., 2003). From the two-back versus control contrast we selected ROIs from the right dorsolateral prefrontal cortex (DLPFC) (MNI coordinates 44, 36, 20 ) and from the right orbital FI (MNI coordinates $38,26,-10)$. To obtain reasonably focal ROIs, we used a conservative threshold for significant clusters of activation determined using the joint expected probability distribution with height $(p<0.0001)$ and extent $(p<0.0001)$ thresholds, corrected at the whole-brain level. This resulted in a 58-voxel right DLPFC ROI and a 36-voxel right orbital FI ROI. These ROIs were then used as seed regions for separate fcMRI analyses (see Fig. 1). That is, after removing the first eight $n$ frames to allow for stabilization of the magnetic field, the average time series from the task-free scan was extracted from the ROI by averaging the time series of all voxels in the ROI. Before averaging individual voxel data, scaling and filtering steps were performed across all brain voxels as follows. To minimize the effect of global drift, voxel intensities were scaled by dividing the value of each time point by the mean value of the whole-brain image at that time point. Next, the scaled waveform of each brain voxel was filtered using a bandpass filter $(\sim 0.0083 / \mathrm{s}<f<\sim 0.15 / \mathrm{s})$ to reduce the effect of lowfrequency drift and high-frequency noise (Lowe et al., 1998). The scaling and filtering steps were applied equivalently to all voxels (including those in the ROIs). The resulting time series, representing the average intensity (after scaling and filtering) of all voxels in the ROI, was then used as a covariate of interest in a whole-brain, linear regression, statistical parametric analysis. As a means of controlling for non-neural noise in the ROI time series (Fox et al., 2005) we included, as nuisance covariates, the time series of two small seven-voxel spherical ROIs created in the white matter of the bilateral frontal lobes. Contrast images corresponding to the ROI time series regressor were derived individually for each subject, and entered into a second-level, random-effects analysis (height and extent thresholds of $p<0.001$ for significant clusters, corrected at the whole brain level) to determine the brain areas that showed significant functional connectivity across subjects.

ICA data. After discarding the first eight $n$ frames to allow for stabilization of the magnetic field, the smoothed images were concatenated across time into a single four-dimensional image. The four-dimensional image was then subjected to ICA with FSL melodic ICA software (www.fmrib.ox.ac.uk/fsl/melodic2/index.html). ICA is a statistical technique that separates a set of signals into independent (uncorrelated and nonGaussian) spatiotemporal components (Beckmann and Smith, 2004). When applied to the $\mathrm{T}^{*}$ signal of fMRI, ICA allows not only for the removal of artifact (McKeown et al., 1998; Quigley et al., 2002), but for the isolation of task-activated neural networks (McKeown et al., 1998; Gu et al., 2001; Calhoun et al., 2002). Most recently, ICA has been used to identify low-frequency neural networks during task-free or cognitively undemanding fMRI scans (Greicius et al., 2004; van de Ven et al., 2004; Beckmann et al., 2005). We allowed the software to estimate the optimal number of components for each scan. Bandpass filtering, helpful in removing high- and low-frequency noise before running ROI analyses, is probably less critical in ICA, which isolates these noise sources as independent components (De Luca et al., 2006). Given the potential risk of removing signal in addition to noise, bandpass filtering was not applied to the data used in the ICA experiments.

The best-fit components for the right DLPFC network and the right FI network were selected in an automated three-step process as in our pre- 
vious studies (Greicius et al., 2004). This process is illustrated in supplemental Figure 1 (available at www.jneurosci.org as supplemental material). First, because intrinsic connectivity is detected in the very lowfrequency range (Cordes et al., 2001), a frequency filter was applied to remove any components in which high-frequency signal $(>0.1 \mathrm{~Hz})$ constituted $50 \%$ or more of the power in the Fourier spectrum. Next, we used the ROI-derived group maps of the right DLPFC and right FI networks from the first group of subjects (see Fig. 1) as standard templates to obtain goodness-of-fit scores for the remaining low-frequency components of each subject. To do this, we used a template-matching procedure that calculates the average $z$-score of voxels falling within the template minus the average $z$-score of voxels outside the template and selects the component in which this difference (the goodness-of-fit) is the greatest. $z$-scores here reflect the degree to which the time series of a given voxel correlates with the time series corresponding to the specific ICA component, scaled by the SD of the error term. The $z$-score is therefore a measure of how many SDs the signal is from the background noise. Finally, the component with the highest goodness-of-fit score is selected as the "best-fit" component and used in the subsequent group analyses. This template-matching procedure was performed separately for each network and, in 18 of 21 subjects, separate components were identified for each network. In three of 21 subjects, this algorithm selected the same component for each network. It is important to note that this approach does not alter the components to fit the template in any way, but merely scores the predetermined components on how well they match the template.

All group analyses were performed on the subjects' best-fit component $z$-score images. We used a random-effects model that estimates the error variance across subjects, rather than across scans (Holmes and Friston, 1998) and therefore provides a stronger generalization to the population from which data are acquired. It should be noted that although the bestfit components were selected with a standard template, the images have $z$-scores assigned to every voxel in the brain so that the group analyses were not constrained by the standard template used to select the components. Using SPM2, one-sample $t$ tests were computed separately to generate group-level maps of the two networks. Significant clusters of activation were determined using the joint expected probability distribution (Poline et al., 1997) with height $(p<0.001)$ and extent $(p<0.001)$ thresholds, corrected at the whole-brain level.

Behavioral correlation analyses (ICA data). To test our hypotheses regarding correlations between network functional connectivity and emotion and cognition, we performed four separate covariate-of-interest analyses to determine whether functional connectivity in either ICAderived network correlated significantly with prescan anxiety or performance on the Trail Making Test (Trails B - Trails A time). These covariate-of-interests analyses were masked to the respective group-level networks, thresholded at $p<0.01$ height and extent, corrected at the whole-brain level. For example, when testing for correlations between prescan anxiety and right FI network functional connectivity, the analysis was limited to those regions included in the group-level right FI network. For these covariate analyses, significant clusters of activation were determined using height and extent thresholds of $p<0.01$, corrected at the whole-brain level.

Post hoc analysis of nodes common to both networks. Although the two networks identified here are mostly nonoverlapping, a few significant clusters were seen in both networks. To explore this phenomenon further, we first identified those voxels that appeared in both networks using either the ROI or ICA approach. These shared nodes were determined by performing an intersection analysis of the four thresholded statistical maps of the networks (the two ROI-derived maps shown in Fig. 1 and the two ICA-derived maps shown in Fig. 2). This analysis produced three small clusters; the largest one, consisting of 21 voxels, was located in the left FI region $(-32,24,-10)$. Then, in the 14 subjects used for the ROI analysis, we examined intrasubject correlations between this left FI cluster and the two seed ROIs (right FI and right DLPFC) used to derive the network maps in Figure 1. Finally, using the ICA data, we calculated intrasubject correlation coefficients for the executive-control and salience network component time series in each subject. For the three of 21 subjects in whom the template-matching algorithm selected the same component for each network, the correlation coefficient was set to 1 .

\section{Results Intrinsic connectivity analyses \\ ROI}

To disentangle the networks evoked by task performance (TAE) we used brain activations elicited during a spatial working memory task (Krasnow et al., 2003) to select two ROIs within the right frontal lobe (Fig. 1A). The first ROI was centered on the right FI (caudal area 47/12 into the anterior insula), chosen for its purported roles in interoceptive and autonomic processing (Craig, 2002; Critchley, 2005). We selected the second ROI from the right dorsolateral PFC (areas 45/46) for its established roles in working memory and control processes (Curtis and D'Esposito, 2003; Kerns et al., 2004). We then determined the functional connectivity of each ROI in a group of 14 subjects scanned during undirected wakefulness with eyes closed. For each subject, the averaged BOLD signal time course from each ROI was used as a regressor to identify brain regions whose BOLD signal fluctuations were highly correlated with the ROI (height and extent thresholds of $p<0.001$ for significant clusters, corrected at the whole-brain level). Using this approach, we separated the TAE into two distinct networks (Fig. $1 B$, supplemental Fig. 2, Table 1, available at www.jneurosci.org as supplemental material). The right FI showed prominent intrinsic connectivity with related paralimbic regions, subcortical and brainstem structures, and the limbic system, whereas right DLPFC connectivity involved primarily lateral frontal-parietal heteromodal association cortices.

\section{ICA}

To replicate and expand on our findings with a convergent method in a larger dataset, network maps of intrinsic connectivity were constructed using ICA in a separate group of 21 subjects. We used an automated template-matching procedure (Greicius et al., 2004) using the right FI and DLPFC networks, identified with the ROI-based analysis, as templates (supplemental Fig. 1, available at www.jneurosci.org as supplemental material). This method allowed us to pinpoint the component for each subject that best corresponded to the right FI and DLPFC networks identified in the ROI analyses. The goodness-of-fit scores, reflecting the spatial correlation between the ROI-derived network template and a subject's best-fit component, are shown for each subject and both networks in supplemental Figure 3 (available at www.jneurosci.org as supplemental material). The ICA approach provided two chief benefits. First, it confirmed that there are two stable and distinct networks anchored by the FI and DLPFC. Second, it uncovered nodes not easily identified with the ROIdriven analysis, which is more prone to contamination by nonneural signals that ICA is able to identify and isolate (Beckmann et al., 2005). Figure 2 (supplemental Fig. 2, Table 2, available at www.jneurosci.org as supplemental material) shows the grouplevel maps of the ICA-derived networks, which reproduce and extend the major findings from the ROI analysis. The ICAderived right FI network is composed of related paralimbic regions, including the bilateral FI, anterior insula, dACC/paracingulate cortex, and the superior temporal pole. There is additional connectivity with the DLPFC (BA 46) and supplementary motor area (SMA)/pre-SMA, as well as frontal, temporal, and parietal opercular regions. Remarkably, this network further includes subcortical sites predicted by the monkey tract tracing literature (Mesulam and Mufson, 1982; Ongur and Price, 2000), including the sublenticular extended amygdala, ventral striatopallidum, dorsomedial thalamus, hypothalamus, periaqueductal gray, and 

Right FI ROI Right
DLPFC ROI
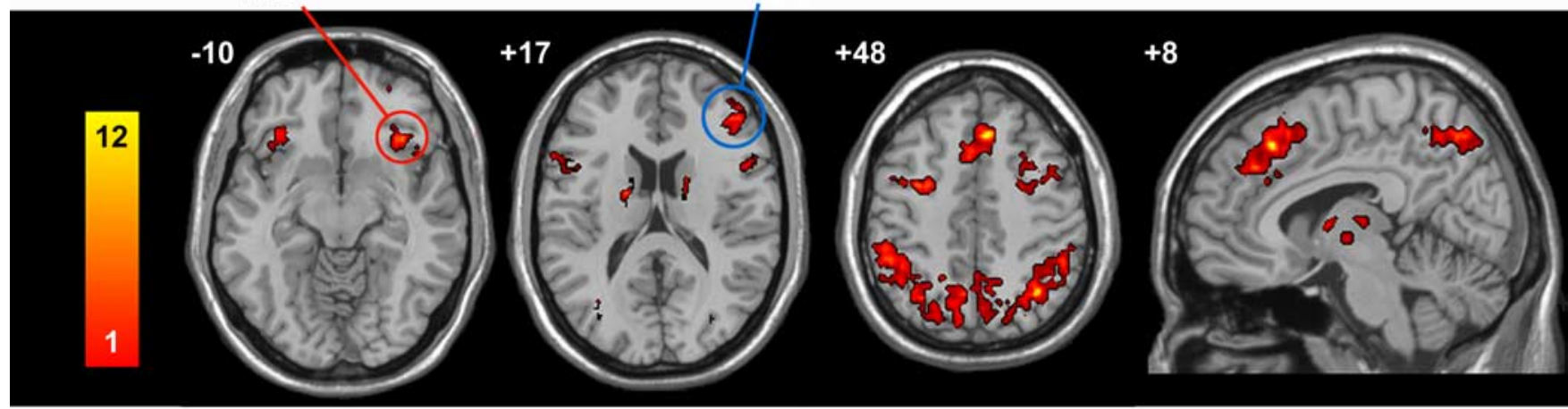

B

ROI functional connectivity maps during undirected wakefulness

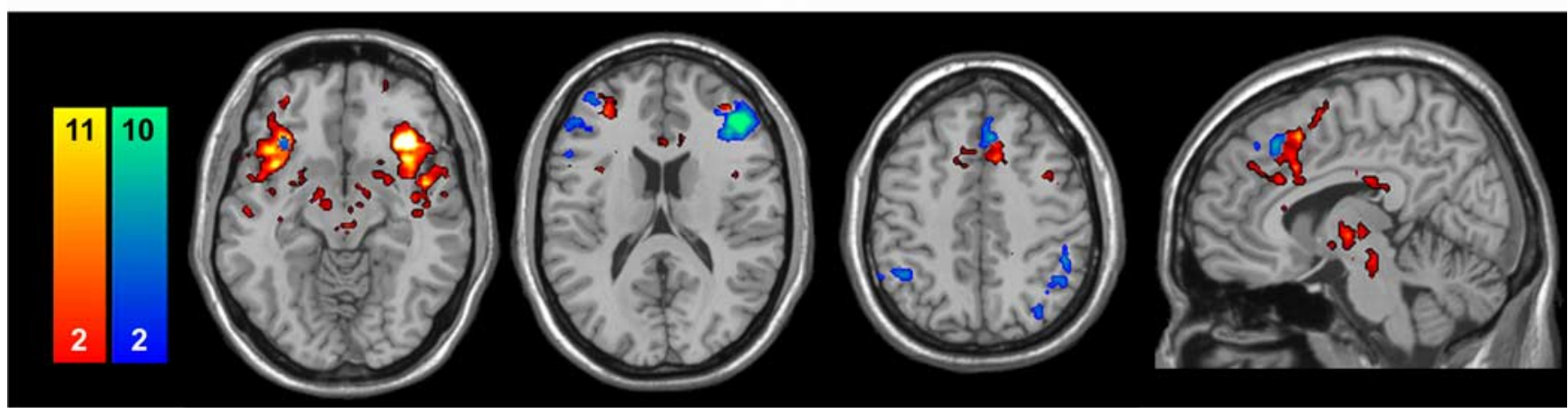

Figure 1. Disentangling the task-activation ensemble with task-free fcMRI. A, A spatial working memory activation map (two-back minus control) was used to select seed R0ls (circled) within the right frontal lobe. $\boldsymbol{B}$, Temporal correlations in BOLD signal determined the intrinsic connectivity patterns with the frontoinsular (red-orange colorbar) and dorsolateral prefrontal (blue-green colorbar) ROls (height and extent thresholds, $p<0.001$, corrected) during undirected wakefulness. For display purposes, the $t$-score color bars in $\boldsymbol{B}$ were adjusted so that the top of the bar reflects the maximum $t$ score seen outside the seed ROI for each network. Effortful tasks like the one used in $\boldsymbol{A}$ often coactivate the networks disentangled using a task-free, intrinsic connectivity analysis in $\boldsymbol{B}$. These ROI-based maps (B) were used as templates for subsequent independent components analyses (Fig. 2). Functional images are displayed on a standard brain template (MNI). On axial and coronal images, the left side of the image corresponds to the left side of the brain.

substantia nigra/ventral tegmental area. We refer to this network, which unites conflict monitoring, interoceptive-autonomic, and reward-processing centers as the "salience network," based on the relevant literature and our behavioral findings detailed subsequently.

The ICA-derived right DLPFC network, in contrast, is made up of lateral neocortical sites involved in cognition. Specifically, the bilateral DLPFC, ventrolateral PFC, dorsomedial PFC, and lateral parietal cortices are incorporated, as well as a site in the left frontoinsula. Consistent with monkey anatomic data (Selemon and Goldman-Rakic, 1988), this group of regions is functionally coupled to the dorsal caudate and anterior thalamus but lacks connectivity with limbic, hypothalamic, and midbrain structures. We refer to this network as the "executive-control network," based on the association of its nodes with working memory and control processes and the behavioral correlations of this study.

Behavioral correlations with intrinsic connectivity networks The most far-reaching question we posed was whether regional functional connectivity within the salience and executive-control networks in the task-free setting would correlate with subject attributes measured outside the scanner. In other words, do individual differences in intrinsic connectivity strength correlate with how one feels and thinks in daily life? We hypothesized that the salience network would relate to interoceptive-autonomic processing, and we asked subjects to rate their level of prescan anxiety using a 10-point VAS. Probing both networks, we sought regions whose functional connectivity in the undirected state was correlated with VAS anxiety ratings. As shown in Figure 3 (supplemental Fig. 2, available at www.jneurosci.org as supplemental material), prescan anxiety correlated significantly (height and extent thresholds of $p<0.01$, corrected at the cluster level across the whole brain) with functional connectivity in two nodes in the salience network: the dACC $(10,34,24 ; 500$ voxels; peak $z$-score, $4.03)$ and the left DLPFC (-32, 44, 16; 111 voxels; peak $z$-score, 4.25). There were no regions in the salience network whose functional connectivity was inversely correlated with anxiety. More importantly, anxiety ratings showed no correlation with functional connectivity in the executive-control network. We then performed a parallel analysis of performance on Trails B, an executive-function task with attentional, working memory, and cognitive control demands. We anticipated an inverse correlation between regional functional connectivity and time required for task completion (reflected by the Trails B minus Trails A time), such that greater functional connectivity would reflect superior (faster) performance. Here, we found a significant inverse correlation between Trails B - Trails A time and two nodes in the 


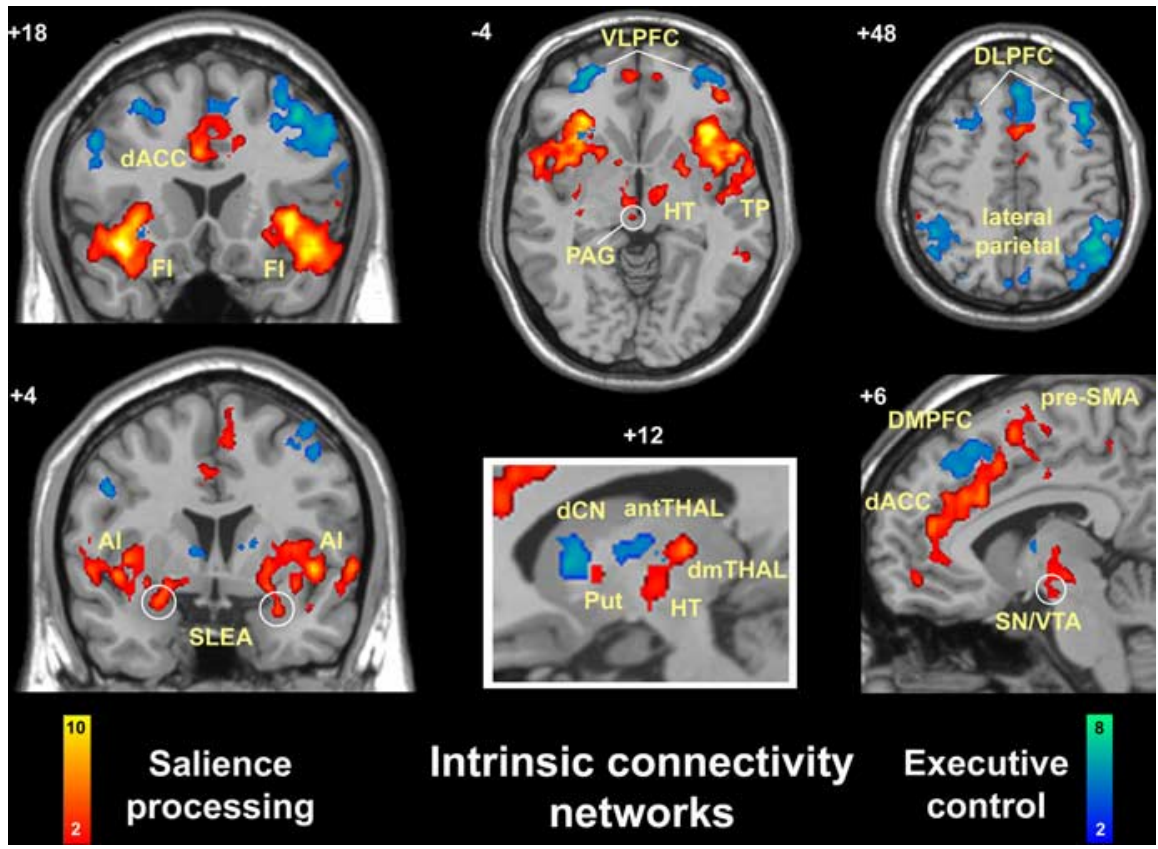

Figure 2. Separable intrinsic connectivity networks revealed by independent component analysis. The salience network (redorange colorbar) is anchored by paralimbic anterior cingulate and frontoinsular cortices and features extensive connectivity with subcortical and limbic structures. In the executive-control network (blue-green colorbar), the dorsolateral frontal and parietal neocortices are linked, with more selective subcortical coupling. Functional images are displayed as in Figure 1. Al, Anterior insula; antTHAL, anterior thalamus; dCN, dorsal caudate nucleus; dmTHAL, dorsomedial thalamus; DMPFC, dorsomedial prefrontal cortex; HT, hypothalamus; PAG, periaqueductal gray; Put, putamen; SLEA, sublenticular extended amygdala; SN/VTA, substantia nigra/ ventral tegmental area; TP, temporal pole; VLPFC, ventrolateral prefrontal cortex.

executive-control network: the left intraparietal sulcus/superior parietal lobule $(-38,-78,36 ; 196$ voxels; peak $z$-score, 4.17$)$ and the right intraparietal sulcus/superior parietal lobule $(36,-80$, 26; 225 voxels; peak $z$-score, 4.05) (Fig. 3, supplemental Fig. 2, available at www.jneurosci.org as supplemental material). There were no regions in the executive-control network whose functional connectivity predicted slower performance, and, critically, Trails B performance did not correlate (positively or negatively) with functional connectivity in the salience network. Thus, intrinsic functional connectivity in the salience network correlated with anxiety but not executive function, whereas intrinsic functional connectivity in the executive-control network correlated with executive function but not anxiety, providing a double dissociation of function between the two networks.

\section{Post hoc analysis of nodes common to both networks}

To identify nodes that were common to both networks, we performed an intersection analysis of the four maps displayed in Figures 1 and 2 (supplemental Fig. 2, available at www.jneurosci.org as supplemental material). This yielded 32 voxels across three clusters, one in the left FI region $(-32,24,-10)$, one in the medial prefrontal cortex $(4,24,48)$, and one in the right DLPFC $(42,48,20)$. We extracted the time series for the left FI ROI (the largest of the three clusters at 21 voxels). Supplemental Table 3 (available at www.jneurosci.org as supplemental material) displays the correlation coefficients between this left FI cluster's time series and the time series from the original seed ROIs (right FI and right DLPFC) for the 14 subjects in the ROI analysis. The mean correlation coefficient between the two seed ROIs was small but significantly different from zero $(r=0.17$; SD, 0.26 ; one-sample $t$ test, $p<0.05)$. The left FI region was significantly correlated with both the right FI region $(r=0.49$; SD, 0.18 ; one- sample $t$ test, $p<0.0001)$ and the right DLPFC ( $r=0.32$; SD, 0.15 ; one-sample $t$ test, $p<0.0001)$. Time series from these three ROIs are shown for two representative subjects in supplemental Figure 4 (available at www.jneurosci.org as supplemental material). Lastly, in supplemental Table 4 (available at www.jneurosci.org as supplemental material), we report the correlation coefficients between the salience component and executive-control component time series in the 21 subjects in the ICA experiment. The mean correlation coefficient here, reflecting the degree of temporal correlation between the networks as a whole, is significantly greater than zero $(r=0.18$; SD, $0.38 ; p<0.05$, one-sample $t$ test), but suggests that temporal activity in one network explains $<5 \%$ of the variance $\left(r^{2}=0.03\right)$ in temporal activity of the other network.

\section{Discussion}

This study forges a new link between intrinsic brain connectivity and how individual brains function. By mapping ICNs during undirected mental activity, we identified two dissociable networks in humans that are critical for guidance of thought and behavior (Ridderinkhof et al., 2004b; Critchley, 2005). These networks, which reflect paralimbic emotional salience processing and dorsal neocortical executive control systems, have been detailed previously in the monkey (Mesulam and Mufson, 1982; Selemon and Goldman-Rakic, 1988; Ongur et al., 1998). Our findings demonstrate the fidelity with which ICNs can extend primate network mapping data to humans. Combined with MR diffusion tractography, ICN mapping may provide a key step toward building a comprehensive human connectivity atlas (Sporns et al., 2005). More importantly, our data show the potential utility of such a project, because ICNs appear to correlate with how humans function outside the scanner.

Interest in ICN mapping has accelerated (Raichle et al., 2001; Greicius et al., 2003; Fox et al., 2005) because of its potential for elucidating brain organization (Beckmann et al., 2005; Fox et al., 2005; Fransson, 2005), function, and dysfunction (Greicius et al., 2004). Key environmental factors during neural development may influence synaptic strengths between spatially distant network components (Bi and Poo, 1999). These strengths, in turn, may bias information processing in a way that influences mental life. In this study, individuals with high dACC connectivity within a salience processing network showed a greater degree of stressor-associated anticipatory anxiety. Likewise, stronger intraparietal sulcus connectivity within an executive-control network predicted superior executive task performance.

\section{Functional significance of the salience and} executive-control networks

The nervous system is continuously bombarded by internal and extrapersonal stimuli. A leading priority is to identify the most homeostatically relevant among these myriad inputs. This capacity requires a system that can integrate highly processed sensory data with visceral, autonomic, and hedonic "markers," 
(Damasio, 1999) so that the organism can decide what to do (or not to do) next. We propose that the salience network described here is well suited for this purpose. It is built around paralimbic structures, most prominently the dACC and orbital frontoinsula, that underlie interoceptiveautonomic processing (Mesulam, 1998; Damasio, 1999; Craig, 2002; Critchley, 2005). These regions coactivate in response to varied forms of salience, including the emotional dimensions of pain (Peyron et al., 2000), empathy for pain (Singer et al., 2004b), metabolic stress, hunger, or pleasurable touch (Craig, 2002), enjoyable "chills" to music (Blood and Zatorre, 2001), faces of loved ones (Bartels and Zeki, 2004) or allies (Singer et al., 2004a), and social rejection (Eisenberger et al., 2003). Most remaining nodes in the salience network are subcortical sites for emotion, homeostatic regulation, and reward (Ongur and Price, 2000; Menon and Levitin, 2005). A thalamic node, which may help bind the circuit together, appears to lie in the dorsomedial nucleus. Although a rough estimation of this ICN has been noted before (Beckmann et al., 2005), here we have detailed its subcortical connectivity for the first time and showed its relationship to individual differences in anxiety state. The findings provide a physiological backdrop for two related research streams, one regarding the cognitive role of the ACC in processing errors and conflict (Menon et al., 2001; Kerns et al., 2004; Ridderinkhof et al., 2004a), and another suggesting that the dACC and FI are specialized modules for sympathetic efference and interoceptive feedback (Critchley et al., 2000, 2004; Craig, 2002; Saper, 2002; Critchley, 2005). In addition to other cytoarchitectonic similarities (Ongur et al., 2003), in humans and great apes the ACC and FI feature a large, bipolar projection neuron found nowhere else in the brain (von Economo, 1926; Nimchinsky et al., 1999). These cells, called von Economo neurons, are far more abundant in humans than in apes and may provide the human salience network with phylogenetically new yet disease-susceptible capabilities (Allman et al., 2005; Seeley et al., 2006).

In contrast to the salience network, the executive-control network is equipped to operate on identified salience. Such operations require directing attention to pertinent stimuli as behavioral choices are weighed against shifting conditions, background homeostatic demands, and context. To achieve this level of response flexibility, the brain must exert control over posterior sensorimotor representations and maintain relevant data in mind until actions are selected. A network geared for this purpose should, and appears to include known sites for sustained attention and working memory (DLPFC, lateral parietal cortex) (Curtis and D'Esposito, 2003), response selection (dorsomedial frontal/pre-SMA) (Lau et al., 2006), and response suppression (ventrolateral prefrontal cortex) (Ridderinkhof et al., 2004b). Subcortical connectivity of the executive-control network mir- rors that seen in the monkey (Selemon and Goldman-Rakic, 1988) and does not extend to autonomic control sites.

\section{Limitations and relationship to previous work}

An important potential limitation of this study is that VAS anxiety scores were acquired before subjects entered the scanner. As such, we cannot be sure whether these scores accurately reflected subjects' degree of anxiety during the scan (state phenomenon) or instead a general tendency toward anxiety (trait phenomenon). This methodological limitation relates to the critical question of whether spontaneous ICN activity reflects ongoing conscious mental activity or a nonconscious means of maintaining canonical networks in a primed state (Raichle, 2006). Evidence for the latter possibility is growing. For example, some simple sensory ICNs are detectable in anesthetized children (Kiviniemi et al., 2000) and even putatively cognitive ICNs appear detectable in subjects presumed to be in the early stages of sleep (Fukunaga et al., 2006). In light of these findings, the correlations reported here suggest that a subject's anxiety trait (rather than state) is coded, to some degree, in the underlying neural architecture of the salience network. Similarly, a subject's cognitive processing and set-shifting speed appears to be coded, to some degree, in the connectivity strength of bilateral intraparietal sulcus nodes of the executive-control network. Additional studies are needed to dissect ICN trait versus state contributions, using validated measures in large cohorts.

This work builds on a series of papers using task-free, intrinsic 
connectivity analyses. Using both ROI-based methods (Biswal et al., 1995; Cordes et al., 2000; Hampson et al., 2002) and ICA (Beckmann et al., 2005; Damoiseaux et al., 2006; De Luca et al., 2006), multiple labs have demonstrated separate ICNs corresponding to the sensorimotor cortex, primary auditory and visual areas, and language centers. Previous work using both ROI analyses (Greicius et al., 2003; Fox et al., 2005; Fransson, 2005; Vincent et al., 2006) and ICA (Greicius and Menon, 2004; Beckmann et al., 2005; Damoiseaux et al., 2006; De Luca et al., 2006) has focused on the ICN known as the default mode network: a set of consistently "deactivated" brain regions whose activity waxes during task-free periods and wanes during task performance (Raichle et al., 2001). In the last two years, ICNs featuring typically activated brain regions (e.g., DLPFC, lateral parietal cortex, anterior cingulate, anterior insula) have also been reported with both ROI-based methods (Fox et al., 2005; Fransson, 2005) and ICA (Beckmann et al., 2005; Damoiseaux et al., 2006; De Luca et al., 2006). The ROI-based papers by Fox et al. (2005) and Fransson (2005) have reported two inversely correlated ICNs referred to as task-positive and task-negative (default mode) networks. The single task-positive network reported in these two papers has consistently been divided into separate networks when ICA is used (Beckmann et al., 2005; Damoiseaux et al., 2006; De Luca et al., 2006). Conflation of these distinct networks in ROI studies seems to result from how the connectivity maps are defined. In the papers by both Fox et al. (2005) and Fransson (2005), the task-positive network is defined in part (Fox et al., 2005) or exclusively (Fransson, 2005) as regions showing inverse correlations with the default mode. A previous report by our group showed that both the right FI and right DLPFC nodes used in the current study are inversely correlated with the posterior cingulate, a prominent region within the default mode network (Greicius et al., 2003). In short, the most ecumenical interpretation of the previous studies and current findings is that in task-free settings both the salience network and the executive-control network are inversely correlated with the default mode network but only minimally correlated with one another.

A most recent study (Hampson et al., 2006), published while the current manuscript was in revision, has also reported a correlation between cognitive function and intrinsic connectivity, although in a separate network. Using an ROI analysis of task-free connectivity in nine subjects, Hampson et al. (2006) reported that greater connectivity between the posterior cingulate and medial prefrontal nodes of the default mode network correlated with better performance on a working memory task. Given the differences in methods, sample sizes, and networks analyzed, it is difficult to compare the results of that study directly with those reported here, except to note that now two independent groups have found correlations between intrinsic connectivity and cognitive performance.

\section{Interactions across ICNs}

Although the thrust of this report has been to demonstrate that the salience and executive-control networks are distinct, it is important, and perhaps informative, to acknowledge that they are not completely independent. We identified three small clusters that were present in both networks (defined with either the ROI or ICA technique). Analysis of activity in the left FI node revealed that it was strongly correlated with both the right FI $(r=0.49)$ and right DLPFC $(r=0.32)$ seed ROIs. In contrast, the component time series (reflecting mean activity across all the nodes in a network) showed only a weak correlation (mean, $r=0.18 ; 21$ subjects) between the networks. These findings suggest that the temporal overlap between the two networks is restricted to a small number of common nodes. Whether such nodes transfer information, facilitate "switching" between networks, or underlie some other key interaction remains an important issue that we hope to pursue in subsequent studies.

In summary, we used fcMRI to detail two fundamental cortical-subcortical networks in the human. These ICNs play vital and dissociable roles in human emotion and cognition. Additional study of ICN profiles may help clarify how differences in neural architecture produce individual differences in thought, feeling, and action patterns.

\section{References}

Allman JM, Watson KK, Tetreault NA, Hakeem AY (2005) Intuition and autism: a possible role for Von Economo neurons. Trends Cogn Sci 9:367-373.

Bartels A, Zeki S (2004) The neural correlates of maternal and romantic love. Neuroimage 21:1155-1166.

Beckmann CF, Smith SM (2004) Probabilistic independent component analysis for functional magnetic resonance imaging. IEEE Trans Med Imaging 23:137-152.

Beckmann CF, DeLuca M, Devlin JT, Smith SM (2005) Investigations into resting-state connectivity using independent component analysis. Philos Trans R Soc Lond B Biol Sci 360:1001-1013.

Bi G, Poo M (1999) Distributed synaptic modification in neural networks induced by patterned stimulation. Nature 401:792-796.

Biswal B, Yetkin FZ, Haughton VM, Hyde JS (1995) Functional connectivity in the motor cortex of resting human brain using echo-planar MRI. Magn Reson Med 34:537-541.

Blood AJ, Zatorre RJ (2001) Intensely pleasurable responses to music correlate with activity in brain regions implicated in reward and emotion. Proc Natl Acad Sci USA 98:11818-11823.

Calhoun VD, Pekar JJ, McGinty VB, Adali T, Watson TD, Pearlson GD (2002) Different activation dynamics in multiple neural systems during simulated driving. Hum Brain Mapp 16:158-167.

Cordes D, Haughton VM, Arfanakis K, Wendt GJ, Turski PA, Moritz CH, Quigley MA, Meyerand ME (2000) Mapping functionally related regions of brain with functional connectivity MR imaging. AJNR Am J Neuroradiol 21:1636-1644.

Cordes D, Haughton VM, Arfanakis K, Carew JD, Turski PA, Moritz CH, Quigley MA, Meyerand ME (2001) Frequencies contributing to functional connectivity in the cerebral cortex in "resting-state" data. AJNR Am J Neuroradiol 22:1326-1333.

Craig AD (2002) How do you feel? Interoception: the sense of the physiological condition of the body. Nat Rev Neurosci 3:655-666.

Critchley HD (2005) Neural mechanisms of autonomic, affective, and cognitive integration. J Comp Neurol 493:154-166.

Critchley HD, Elliott R, Mathias CJ, Dolan RJ (2000) Neural activity relating to generation and representation of galvanic skin conductance responses: a functional magnetic resonance imaging study. J Neurosci 20:3033-3040.

Critchley HD, Wiens S, Rotshtein P, Ohman A, Dolan RJ (2004) Neural systems supporting interoceptive awareness. Nat Neurosci 7:189-195.

Curtis CE, D’Esposito M (2003) Persistent activity in the prefrontal cortex during working memory. Trends Cogn Sci 7:415-423.

Damasio AR (1999) The feeling of what happens: body and emotion in the making of consciousness. Orlando, FL: Harcourt.

Damoiseaux JS, Rombouts SA, Barkhof F, Scheltens P, Stam CJ, Smith SM, Beckmann CF (2006) Consistent resting-state networks across healthy subjects. Proc Natl Acad Sci USA 103:13848-13853.

De Luca M, Beckmann CF, De Stefano N, Matthews PM, Smith SM (2006) fMRI resting state networks define distinct modes of long-distance interactions in the human brain. Neuroimage 29:1359-1367.

Eisenberger NI, Lieberman MD, Williams KD (2003) Does rejection hurt? An FMRI study of social exclusion. Science 302:290-292.

Fox MD, Snyder AZ, Vincent JL, Corbetta M, Van Essen DC, Raichle ME (2005) The human brain is intrinsically organized into dynamic, anticorrelated functional networks. Proc Natl Acad Sci USA 102:9673-9678.

Fransson P (2005) Spontaneous low-frequency BOLD signal fluctuations: an $\mathrm{fMRI}$ investigation of the resting-state default mode of brain function hypothesis. Hum Brain Mapp 26:15-29.

Friston KJ, Ashburner J, Frith CD, Poline JB, Heather JD, Frackowiak RSD 
(1995) Spatial registration and normalization of images. Hum Brain Mapp 2:165-189.

Friston KJ, Price CJ, Fletcher P, Moore C, Frackowiak RS, Dolan RJ (1996) The trouble with cognitive subtraction. Neuroimage 4:97-104.

Fukunaga M, Horovitz SG, van Gelderen P, de Zwart JA, Jansma JM, Ikonomidou VN, Chu R, Deckers RH, Leopold DA, Duyn JH (2006) Largeamplitude, spatially correlated fluctuations in BOLD fMRI signals during extended rest and early sleep stages. Magn Reson Imaging 24:979-992.

Glover GH, Lai S (1998) Self-navigated spiral fMRI: interleaved versus single-shot. Magn Reson Med 39:361-368.

Greicius MD, Menon V (2004) Default-mode activity during a passive sensory task: uncoupled from deactivation but impacting activation. J Cogn Neurosci 16:1484-1492.

Greicius MD, Krasnow B, Reiss AL, Menon V (2003) Functional connectivity in the resting brain: a network analysis of the default mode hypothesis. Proc Natl Acad Sci USA 100:253-258.

Greicius MD, Srivastava G, Reiss AL, Menon V (2004) Default-mode network activity distinguishes Alzheimer's disease from healthy aging: evidence from functional MRI. Proc Natl Acad Sci USA 101:4637-4642.

Grinband J, Hirsch J, Ferrera VP (2006) A neural representation of categorization uncertainty in the human brain. Neuron 49:757-763.

Gu H, Engelien W, Feng H, Silbersweig DA, Stern E, Yang Y (2001) Mapping transient, randomly occurring neuropsychological events using independent component analysis. Neuroimage 14:1432-1443.

Hampson M, Peterson BS, Skudlarski P, Gatenby JC, Gore JC (2002) Detection of functional connectivity using temporal correlations in MR images. Hum Brain Mapp 15:247-262.

Hampson M, Driesen NR, Skudlarski P, Gore JC, Constable RT (2006) Brain connectivity related to working memory performance. J Neurosci 26:13338-13343.

Holmes AP, Friston KJ (1998) Generalisability, random effects and population inference. Neuroimage 7:S754.

Kerns JG, Cohen JD, MacDonald III AW, Cho RY, Stenger VA, Carter CS (2004) Anterior cingulate conflict monitoring and adjustments in control. Science 303:1023-1026.

Kim DH, Adalsteinsson E, Glover GH, Spielman S (2000) SVD regularization algorithm for improved high-order shimming. In: Proceeedings of the 8th Annual Meeting of ISMRM, p 1685. Denver: ISMRM.

Kiviniemi V, Jauhiainen J, Tervonen O, Paakko E, Oikarinen J, Vainionpaa V, Rantala H, Biswal B (2000) Slow vasomotor fluctuation in fMRI of anesthetized child brain. Magn Reson Med 44:373-378.

Krasnow B, Tamm L, Greicius MD, Yang TT, Glover GH, Reiss AL, Menon V (2003) Comparison of fMRI activation at 3 and $1.5 \mathrm{~T}$ during perceptual, cognitive, and affective processing. Neuroimage 18:813-826.

Lau H, Rogers RD, Passingham RE (2006) Dissociating response selection and conflict in the medial frontal surface. Neuroimage 29:446-451.

Lowe MJ, Mock BJ, Sorenson JA (1998) Functional connectivity in single and multislice echoplanar imaging using resting-state fluctuations. Neuroimage 7:119-132.

McKeown MJ, Jung TP, Makeig S, Brown G, Kindermann SS, Lee TW, Sejnowski TJ (1998) Spatially independent activity patterns in functional MRI data during the stroop color-naming task. Proc Natl Acad Sci USA 95:803-810.

Menon V, Levitin DJ (2005) The rewards of music listening: response and physiological connectivity of the mesolimbic system. Neuroimage 28:175-184

Menon V, Adleman NE, White CD, Glover GH, Reiss AL (2001) Errorrelated brain activation during a Go/NoGo response inhibition task. Hum Brain Mapp 12:131-143.

Mesulam MM (1998) From sensation to cognition. Brain 121:1013-1052.
Mesulam MM, Mufson EJ (1982) Insula of the old world monkey. III: efferent cortical output and comments on function. J Comp Neurol 212:38-52.

Nimchinsky EA, Gilissen E, Allman JM, Perl DP, Erwin JM, Hof PR (1999) A neuronal morphologic type unique to humans and great apes. Proc Natl Acad Sci USA 96:5268-5273.

Ongur D, Price JL (2000) The organization of networks within the orbital and medial prefrontal cortex of rats, monkeys, and humans. Cereb Cortex 10:206-219.

Ongur D, An X, Price JL (1998) Prefrontal cortical projections to the hypothalamus in macaque monkeys. J Comp Neurol 401:480-505.

Ongur D, Ferry AT, Price JL (2003) Architectonic subdivision of the human orbital and medial prefrontal cortex. J Comp Neurol 460:425-449.

Peyron R, Laurent B, Garcia-Larrea L (2000) Functional imaging of brain responses to pain. A review and meta-analysis (2000). Neurophysiol Clin 30:263-288.

Poline JB, Worsley KJ, Evans AC, Friston KJ (1997) Combining spatial extent and peak intensity to test for activations in functional imaging. Neuroimage 5:83-96.

Quigley MA, Haughton VM, Carew J, Cordes D, Moritz CH, Meyerand ME (2002) Comparison of independent component analysis and conventional hypothesis-driven analysis for clinical functional MR image processing. AJNR Am J Neuroradiol 23:49-58.

Raichle ME (2006) Neuroscience. The brain's dark energy. Science 314:1249-1250.

Raichle ME, MacLeod AM, Snyder AZ, Powers WJ, Gusnard DA, Shulman GL (2001) A default mode of brain function. Proc Natl Acad Sci USA 98:676-682.

Ridderinkhof KR, Ullsperger M, Crone EA, Nieuwenhuis S (2004a) The role of the medial frontal cortex in cognitive control. Science 306:443-447.

Ridderinkhof KR, van den Wildenberg WP, Segalowitz SJ, Carter CS (2004b) Neurocognitive mechanisms of cognitive control: the role of prefrontal cortex in action selection, response inhibition, performance monitoring, and reward-based learning. Brain Cogn 56:129-140.

Saper CB (2002) The central autonomic nervous system: conscious visceral perception and autonomic pattern generation. Annu Rev Neurosci 25:433-469.

Seeley WW, Carlin DA, Allman JM, Macedo MN, Bush C, Miller BL, Dearmond SJ (2006) Early frontotemporal dementia targets neurons unique to apes and humans. Ann Neurol 60:660-667.

Selemon LD, Goldman-Rakic PS (1988) Common cortical and subcortical targets of the dorsolateral prefrontal and posterior parietal cortices in the rhesus monkey: evidence for a distributed neural network subserving spatially guided behavior. J Neurosci 8:4049-4068.

Singer T, Kiebel SJ, Winston JS, Dolan RJ, Frith CD (2004a) Brain responses to the acquired moral status of faces. Neuron 41:653-662.

Singer T, Seymour B, O’Doherty J, Kaube H, Dolan RJ, Frith CD (2004b) Empathy for pain involves the affective but not sensory components of pain. Science 303:1157-1162.

Sporns O, Tononi G, Kotter R (2005) The human connectome: a structural description of the human brain. PLoS Comput Biol 1:e42.

van de Ven V, Formisano E, Prvulovic D, Roeder CH, Linden DE (2004) Functional connectivity as revealed by spatial independent component analysis of fMRI measurements during rest. Hum Brain Mapp 22:165-178.

Vincent JL, Snyder AZ, Fox MD, Shannon BJ, Andrews JR, Raichle ME, Buckner RL (2006) Coherent spontaneous activity identifies a hippocampal-parietal memory network. J Neurophysiol 96:3517-3531.

von Economo C (1926) Eine neue art spezialzellen des lobus cinguli und lobus insulae. Z Ges Neurol Psychiatr 100:706-712. 